\title{
Case Reports: Contribution to Clinical Practice, Scholarly Education, and Scientific Knowledge
}

\author{
Mahgoub N
}

Department of Psychiatry, Cornell University, White Plains, NY, USA

*Corresponding author: Mahgoub N, Assistant Professor of Clinical Psychiatry, Department of Psychiatry, Cornell University, 21 Bloomingdale Rd, White Plains, NY, USA 10605, E-mail: namd06@aol.com

Citation: Mahgoub N (2017) Case Reports: Contribution to Clinical Practice, Scholarly Education, and Scientific Knowledge. J Case Rep Stud 5(2): 201. doi: 10.15744/2348-9820.5.201

Received Date: January 18, 2017 Accepted Date: April 25, 2017 Published Date: April 27, 2017

\begin{abstract}
Case reports have been a method of scientific communication in the medical literature. Cases describing a clinical challenge faced by physicians and providing well-reasoned recommendations serve an important function in the clinical practice. Other values of case reports include generating clinical hypotheses and promoting scholarly education. They enhance critical thinking for practicing physicians, residents, and medical students. A case report can raise conceptual questions about particular clinical observation with the goal to generate a clinical hypothesis. This article highlights the value of case reports and describes their role in advancement of medicine with the intent to increase and improve case reporting.
\end{abstract}

Keywords: Evidence-Based Practice; Function of Case Reports; How to Publish a Case Report

\section{Introduction}

Case reports are the earliest form of medical communication and serve as a platform to circulate new knowledge gained in clinical practice. From Freud's case report of Anna O illustrating the concept of hysteria in 1895 [1] and McBride's case report of congenital abnormalities of Thalidomide in 1961 [2] to today's medical literature, case reports have been unfolding enormous clinical complexities that are undetectable in clinical trials and bringing new scientific discoveries to attention. It is important to recognize the value of case report in advancing medicine through generating new hypotheses and enhancing scholarly education. Moreover, case reports are considered as the first-line evidence in scientific knowledge as they present original observations that often stimulate research.

This article highlights the value of case reports and describes their role in advancement of medicine with the intent to increase and improve case reporting.

\section{The Relevance of Case Reports in the Era of Evidence-Based Practice}

The strength of evidence in case reports lies in detecting novelty and individualizing interventions. Vandenbroucke 2001 emphasized the high sensitivity of case reports for detecting novelty [3]. Physicians often assess various diseases based on the common pattern of signs or symptoms of the disease. The recognition of new or different patterns of clinical presentation emboldens the case reports [4]. Case reports usually describe new clinical findings and distinctive observations such as atypical presentations of a common disease, unusual side effects to a treatment, or new therapeutic approach to a common condition.

The other feature of case reports is to individualize treatment according to clinical complexity. Evidence-based medicine utilizes the hierarchy classification of evidence to support a therapeutic intervention [5]. The strength and validity of the clinical research determine the effectiveness of the evidence [6]. Accordingly, randomized controlled studies and meta-analyses play a major role in evaluating therapeutic efficacy but they are inadequate when evaluation extends beyond effectiveness and often too general to address the complexity of an individual case. Practice guidelines suggest interventions to be implemented but case reports illustrate the implementation of an intervention. Evidence for the effectiveness of certain intervention alone may not be adequate to guide clinical decisions. Expansion of the evidence to include methods of implementation of interventions is necessary. In this context, case reports play a vital role in guiding the personalization of a treatment in clinical practice. A case report can provide descriptive explanation how particular intervention can be successfully implemented to a particular patient to achieve desirable outcomes. 


\section{Understanding the Function of Case Reports \\ The Role of Case Reports in Generating Clinical Hypotheses}

The value of case reports appears in their contribution to hypothesis generation, such as new disease pathology, new risk factor for an existing disease, and new effect of a pharmacological agent. Case reports represent an example to bring clinicians to bear in the process of formulating conceptual hypotheses and promote critical thinking in clinical practice. The authors need to utilize tools such as fundamental clinical knowledge, basic science, decisional analysis, scientific reasoning, and testing a hypothesis in order to make a case report valuable part of the knowledge continuum.

Most classical biological hypotheses of psychiatry are based on clinical observation of cases. For example, the discovery of chlorpromazine as an antipsychotic has been labeled as one of the great advances in the history of psychiatry [7]. In 1950, a French chemist synthesized a series of compounds including chlorpromazine to produce pronounced sedation in surgical patients. On January 19, 1952, it was administered (along with ECT) to a 24-year-old manic patient, who responded dramatically. Subsequent treatment with chlorpromazine went beyond sedation showing improvements in thinking and emotional behavior. This observation stimulated research studies which revealed chlorpromazine's ability to block dopamine receptors. By 1954, chlorpromazine was being used in the United States to treat manic and psychotic disorders [7].

\section{The Role of Case Reports in Clinical Research}

Case reports may serve as the early phase of clinical research and provide stimulus and new direction for further research. Case reports in a high impact journal can have considerable influence on the literature and research. For example, Albrecht J et al. 2005 [8] identified 64 case reports and 39 small case series published in The Lancet between January 1996 and June 1997. Of the 64 reports 11 (17\%) were followed by clinical trials, 4 (6\%) of which were found in the Register of Current Controlled Clinical Trials. Small case series were followed by trials 13 times (33\%); 5 (13\%) of which were found in the Current Controlled Clinical Trials Register.

Furthermore, a meta-analysis of case reports from the literature can conclude meaningful new findings and lead to research [9]. For example, meta-analysis of case reports of ischemic colitis in young patients revealed that the disease seems to be of young women taking oral contraceptives; this finding was confirmed later in a case-control study [9].

\section{The Role of Case Reports in the Scholarly Practice of Medicine}

Case reports have been central to providing a mechanism for scholarly education and advancing clinical knowledge. They serve as important educational tools for training academic psychiatrists, junior faculty, medical students, residents and fellows in critical thinking and scientific writing.

Case reports offer a structure for case-based learning in medical education [10]. The educational value becomes apparent when the physician discusses his diagnostic or therapeutic reasoning of the case. This discussion is imperative in improving physician's clinical knowledge through enhancing awareness of rare disorders to facilitate diagnosis, clarifying new aspects on the etiology of a disorder, or explaining indistinct treatment response. Moreover, a case presentation can enhance critical reasoning skills through formulating a clinical question, finding the best evidence, appraising the evidence, and applying the evidence to the patient [10]. Conceptual hypothesis can be subsequently generated from the case presentation and published as a case report.

Given that case reports are viewed as the first-line of evidence, by writing case reports, medical students and residents can contribute to the first line of evidence for developing new treatment. Moreover, case reports can be an excellent way for medical students and residents to get started with academic writing [10]. They will learn how to collect data from various resources, perform a literature review, structure a manuscript, obtain consent from the patient, and how to submit and revise the manuscript.

\section{The Influence of Case Reports on Direct Patient Care}

Case reports can contribute to a change in clinical practice. They enable physicians to share their retrospective experiences and challenges with individual cases, and sensitize other physicians to similar cases. They can provide some guidance for conditions that do not require randomized trials such as uncommon presentations [11]. Moreover, case reports can give some directions for conditions that cannot wait for trials to be conducted such in emerging infectious diseases [12].

Case reporting remains important for detection of side effects of drugs, whether adverse or beneficial [3]. For example: Sildenafil was developed from observation of a side effect of an antihypertensive medication [13], and the observation that the nicotine withdrawal symptoms were linked to depression directed the use of certain antidepressant medications as smoking cessation agents [14]. Furthermore, case reports have a potential role in recognizing the risk factors of an illness. For example, the first case report of aplastic anemia by Ehrlich in 1888 was in a young pregnant woman. Since then, pregnancy has been included in the list of potential causes of aplastic anemia [15]. 


\section{How to Translate Clinical Experience into a Publishable Material}

Writing a clinical topic is a structured process in which the physician describes a noteworthy clinical observation and his critical reasoning. This process should fulfill the need for accuracy, precision, and intelligibility through well-constructed text. Case reports often serve as the physician's first experience with scholarly writing and provide an important training ground in manuscript preparation and publication. The case is determined to be reportable based on novelty and educational value.

It is important to differentiate evidence-based case report from the traditional case report, which is a recollective description of a clinical case and comparing it with the existing knowledge. Whereas evidence-based case report documents how research evidence has been applied to guide the treatment of a particular case and evaluate the clinical outcome. Traditional case reports usually describe innovations but evidence-based case reports are intended to demonstrate a process [16].

A traditional case report is the scientific documentation of a clinical observation that illustrates diagnostic or therapeutic challenge or describes new clinical findings [17]. Strengths and weaknesses of a case report depend on the structure and content of the report in terms of building a clinical question, structured presentation, convincing argument, scientific inference, substantiated conclusion, and valid recommendation. Good case reporting demands a clear focus to make explicit to the readers why a particular clinical observation is important in the context of existing knowledge. Another important rule in writing good case report is to convey one single message that would be the primary focus of the report [16].

An evidence-based case report should illustrate the evidence-based approach as follows: asking answerable clinical questions, searching for the evidence, critically appraising the evidence for its validity and relevance, making a decision by integrating the evidence with clinical expertise, and evaluating the outcome [18]. Moreover, an evidence-based case report should show how evidence can be applied at all stages of patient care. For example, decisions about which tests to order can be guided by information on the sensitivity and specificity of different tests. Decisions about which interventions to advise can be informed by randomized controlled trials and systematic reviews comparing effectiveness and safety. Information on side effects can be concluded from well designed cohort or case control studies. When the physician fails to achieve desirable outcomes, he critically highlights the gap in the evidence and generates clinical hypotheses [16].

\section{Conclusion}

The practice of medicine involves integrating individual clinical experience with the best available evidence from the medical literature. Case reporting in the era of clinical trials and systematic reviews puts a considerable weight on categorizing clinical evidence, particularly with the increasing importance of individualized care. Moreover, case reports can contribute to the scientific knowledge through enhancing hypotheses generation and scholarly education [19]. Finally, writing an informative case report requires practicing, inquiring others who published, pairing clinicians with academicians, socializing in a scholarly environment, and creating a forum for a functional dialogue between physicians and researchers [20].

\section{References}

1. Castelnuovo-Tedesco P (1994) On rereading the case of Anna O.: more about questions that are unanswerable. J Am Acad Psychoanal 22: 57-71.

2. McBride WG (1961) Thalidomide Case Report. The Lancet 2: 1358.

3. Vandenbroucke JP (2001) In defense of case reports and case series. Ann Intern Med 134: 330-4.

4. Coentre R, Silva-Dos-Santos A, Talina MC (2016) Acute Psychosis as Major Clinical Presentation of Legionnaires' Disease. Case Rep Psychiatry $10.1155 / 2016 / 3519396$.

5. Evans D (2003) Hierarchy of evidence: a framework for ranking evidence evaluating healthcare interventions. J Clin Nurs $12: 77-84$.

6. Burns PB, Rohrich RJ, Chung KC (2011) The levels of evidence and their role in evidence-based medicine. Plast Reconstr Surg 128: 305-10.

7. López-Muñoz F, Alamo C, Cuenca E, Shen WW, Clervoy P, et al. (2005) History of the discovery and clinical introduction of chlorpromazine. Ann Clin Psychiatry 17: 113-35.

8. Albrecht J, Meves A, Bigby M (2005) Case reports and case series from Lancet had significant impact on medical literature. J Clin Epidemiol 58: 1227-32.

9. Deana DG, Dean PJ (1995) Reversible ischemic colitis in young women. Association with oral contraceptive use. Am J Surg Pathol 19: 454-62.

10. Florek AG, Dellavalle RP (2016) Case reports in medical education: a platform for training medical students, residents, and fellows in scientific writing and critical thinking. J Med Case Rep 10: 86.

11. Nakamura T, Igarashi H, Ito T, Jensen RT (2014) Important of case-reports/series, in rare diseases: Using neuroendocrine tumors as an example. World J Clin Cases 2: 608-13.

12. Wiwanitkit V (2011) The usefulness of case reports in managing emerging infectious disease. J Med Case Rep 5: 194.

13. Jenicek M (1999) Clinical Case Reporting in Evidence-Based Medicine. Oxford: Butterworth-Heinemann, USA.

14. Covey LS, Sullivan MA, Johnston JA, Glassman AH, Robinson MD, et al. (2000) Advances in non-nicotine pharmacotherapy for smoking cessation. Drugs 59: 17-31.

15. Oosterkamp HM, Brand A, Kluin-Nelemans JC, Vandenbroucke JP (1998) Pregnancy and severe aplastic anaemia: causal relation or coincidence? Br J Haematol 103: 315-6.

16. Godlee F (1998) Applying research evidence to individual patients. Evidence-based case reports will help. BMJ 316: 1621-2.

17. Kotbi N, Mahgoub N, Mokonogho J, Young R (2009) Rhabdomyolysis associated with mania in late life. Int J Geriatr Psychiatry 24 : 1478-9. 
18. Jones-Harris AR (2003) The evidence-based case report: a resource pack for chiropractors. Clinical Chiropractic 6: 73-84.

19. Neely JG, Karni RJ, Nussenbaum B, Paniello RC, Fraley PL, et al. (2008) Practical guide to understanding the value of case reports. Otolaryngol Head Neck Surg 138: 261-4.

20. Mahgoub NA, Alexopoulos GS (2010) Bringing clinicians to bear in the hypothesis generation process. Acad Psychiatry 34: 241-2.

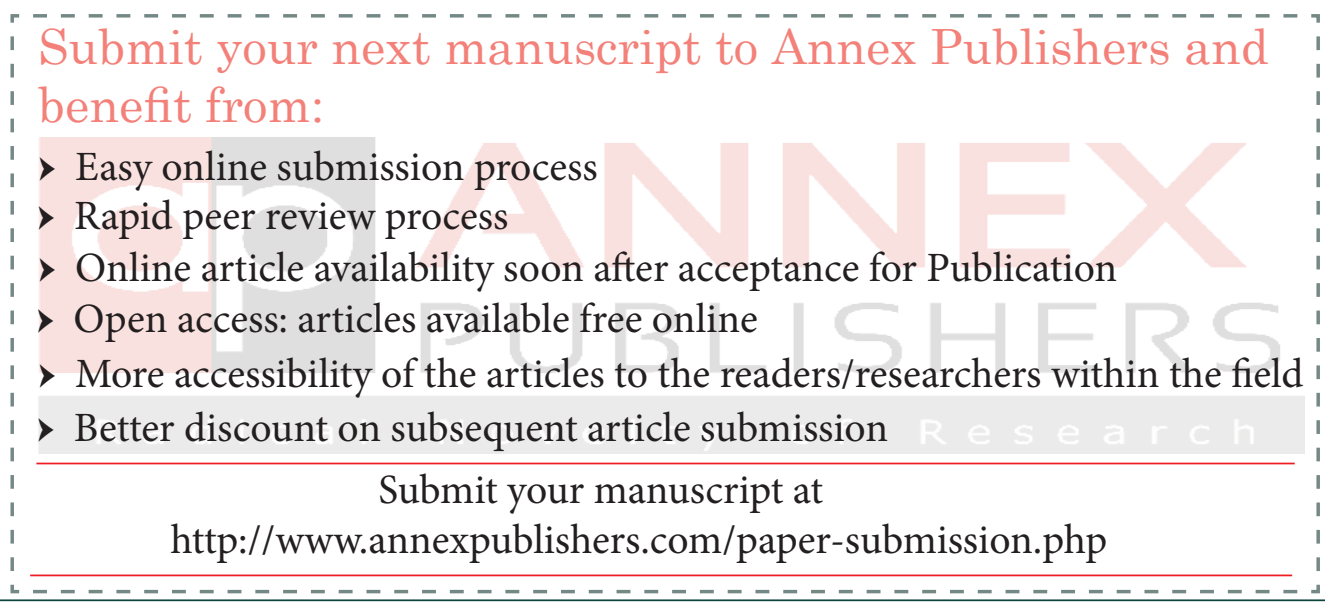

